Accepted manuscript (c) <2020>. This manuscript version is made available under the CC-BY-NC-ND 4.0 license http://creativecommons.org/licenses/by-nc-nd/4.0/

Publication: J. Synchrotron Radiation 26, 1929 (2019)

\title{
Gold nano-mesh synthesis by continuous-flow X-ray irradiation
}

\author{
Min-Tsang Li, ${ }^{a, b}$ Sheng-Feng Lai, ${ }^{b}$ Shun-Min Yang, ${ }^{b}$ Yu-Sheng Chen, ${ }^{b}$ Ying-Jie Chen, ${ }^{b}$ Eng \\ Soon Tok, ${ }^{c}$ Giorgio Margaritondo ${ }^{d}$ and Yeukuang Hwu ${ }^{b, a}$ \\ a Department of Engineering Science, National Cheng Kung University, Tainan 70101, Taiwan, \\ b Institute of Physics, Academia Sinica, Taipei 11529, Taiwan, \\ ${ }^{c}$ Department of Physics, National University of Singapore, Singapore 119077, Singapore \\ d Ecole Polytechnique Fédérale de Lausanne (EPFL), Lausanne 1015, Switzerland.
}

\begin{abstract}
X-ray irradiation has been extensively used in recent years as a fabrication step for nanoparticles and nanoparticle systems. A variant of this technique, continuous-flow X-ray irradiation, has recently been developed, and offers three important advantages: precise control of the irradiation dose, elimination of convection effects in the precursor solution, and suitability for large-scale production. Here, the use of this method to fabricate Au nanomeshes of interest as transparent and flexible electrodes for optoelectronics is reported. The study includes extensive characterization of the synthesis parameters and of the product properties, with rather encouraging results.
\end{abstract}

\section{Introduction}

The growth of nanotechnology systems requires a constant development of new fabrication techniques. In particular, methods based on X-ray irradiation have produced many positive results in recent years. However, such methods are still affected by problems such as the reliable control of fabrication parameters and the use for mass production.

We are now developing a special variety of irradiation growth of nanosystems, in which the precursor solution is forced to flow at a controlled rate in front of the irradiating beam. The flow rate determines the total irradiation dose, which can thus be controlled easily and with great accuracy. This is an important achievement, since the dose is a crucial factor in the final characteristics of the products.

We present here some of the first results of this 'continuous- flow X-ray irradiation' method, specifically the fabrication of accurate $A u$ meshes with nanoscale structures, good conductivity, flexibility and transparency to visible light. Such properties make them suitable for flexible optoelectronics devices and therefore very interesting for technology.

Specifically, our products can be used as transparent conducting electrodes (TCEs), which are key components of optoelectronics devices such as touchscreens, e-papers, organic light-emitting diodes (OLEDs), liquid-crystal displays and solar cells. They could replace in flexible devices tin- doped indium oxide (ITO) and fluorine-doped tin oxide electrodes, with several advantages (Edwards et al., 2004) such as their non-brittle nature (Chen et al., 2002). They could also become strong competitors for Ag nanowires, the alternate solution 
for flexible optoelectronics, which is affected by a series of problems such as long-term instability (Wu et al., 2004; Gou et al., 2007; Kang \& Guo, 2007; Eda et al., 2008; Lee et al., 2008; De et al., 2009; Doherty et al., 2009; Reina et al., 2009; Tung et al., 2009; Catrysse \& Fan, 2010; Hecht et al., 2011; Khaligh \& Goldthorpe, 2013; Cheon et al., 2017; Zhang et al., 2017).

Our present work is built on many years of research on nanofabrication by X-ray irradiation. We specifically applied this approach to naked and polymer-coated metallic nano- particles (Wang et al., 2007, 2008, 2009), bimetallic nanoparticles (Wang et al., 2011; Li et al., 2015) and fluorescent gold nanoparticles (Lai et al., 2013, 2015). Fabrication by irradiation offers superior advantages including a fast and simple control of the nanoparticle size.

These above studies also revealed and empirically investigated a wealth of physical mechanisms underlying the irradiation processes. With intense $X$-rays bombarding uniformly the entire aqueous solution, strong reducing agents, such as $\mathrm{H}^{\cdot}$ radicals and hydrated electrons $\left(e^{-}\right.$aq $)$, were generated almost instantly and converted metallic ions into metal atoms. The nuclei formed at supersaturated metallic atoms, then grew to larger particles. This process continued until the solution was depleted of metallic atoms. The important point is that such multiple mechanisms enhance the actual and potential flexibility in controlling the synthesis parameters and the products. However, only a fraction of this flexibility has been exploited so far.

Specifically, the previous synthesis procedures used individual batches of precursors loaded in tubes and then exposed to X-rays (Wang et al., 2007; Liu et al., 2009). The radiation exposure time was controlled by the X-ray shutter. In the initial phase of parameter optimization, the convection in the precursor solution limited the processes to small volumes and short exposures (Yang et al., 2006). This approach cannot be used for large volumes: the $\mathrm{X}$-ray attenuation requires longer irradiations, and due to convection the irradiation time cannot control the exposure. This is a problem, since accurate expo-sure control is critical in many cases, for example in the synthesis of MUA-Au nanoparticles to produce nanoparticles of homogeneous small size and with strong photoluminance (Lai et al., 2012). The new step in continuous-flow X-ray irradiation is motivated by several factors. First, the total irradiation is determined by the time spent by the precursor solution in front of the $X$ ray beam. This simply corresponds to the flow rate, so that by tuning the rate we can accurately control the irradiation. In turn, we previously demonstrated that the irradiation determines a series of important product parameters such as the nanoparticle size and shape.

This variant of X-ray nanofabrication can be readily used for mass production with accurate control of the products. We investigated the corresponding issues by studying the effects not only of the flow rate but also of the capping agent and of the $\mathrm{pH}$ of the precursor solution.

With a specific set of fabrication conditions, the synthesized Au nanoparticles form twodimensional random net structures material on several different substrates, such as $\mathrm{Si}$ and glass, without requiring any additional treatment. The size of our Au nano-meshes is up to several micrometers and the average nanoparticle diameter is $\approx 10 \mathrm{~nm}$.

Some important properties of the Au nano-meshes were measured, finding results suitable for transparent conductive films. Overall, our findings are quite encouraging and show that continuous-flow $X$-ray irradiation is a simple and effective technique for the mass production 
of important optoelectronic device components. Its applications could potentially extend to other devices and other domains.

\section{Materials and methods}

$\mathrm{HAuCl}_{4} \cdot 3 \mathrm{H}_{2} \mathrm{O}$, sodium hydroxide, (hydroxypropyl)methyl cellulose (HPMC) (viscosity 2600$5600 \mathrm{cP}$ ), poly(ethyl glycol) 6000 and 20000, polyvinylpyrrolidone 40000, poly(vinyl) alcohol 130000 , polyethylenmine (branched) 2000, poly-(acrylic acid) 15000, citric acid and dimethyl sulfoxide (DMSO) were purchased from Sigma-Aldrich. All chemicals were reagent grade. Distilled deionized (d.d.) water was purified with a Millipore Milli-Q water system. The aqueous precursor solution was prepared by mixing $\mathrm{HAuCl}_{4} \cdot 3 \mathrm{H}_{2} \mathrm{O}(10 \mathrm{mM})$ and adjusting the $\mathrm{pH}$ with sodium hydroxide $(0.1 \mathrm{M})$ to reach the final $\mathrm{Au}$ concentration of $1 \mathrm{mM}$.

The continuous flow through the X-ray beam was implemented by pumping the precursor solution with an adjustable- speed rotary pump into a stainless steel convection-free reactor with a $11.5 \mathrm{~mm} \times 7 \mathrm{~mm} \times 0.5 \mathrm{~mm}$ reaction chamber. The reactor had a Kapton X-raytransmitting front window connecting it to the BL01A beamline of the National Synchrotron Radiation Research Center (NSRRC, Hsinchu, Taiwan), as well as a back window allowing the X-ray beam to pass through. Details of the X-ray source have been reported elsewhere (Wang et al., 2007; Liu et al., 2009; Song et al., 2007).

Note that the BL01A beamline uses a superconducting wavelength shifter to produce highenergy photons from a medium-energy, $1.5 \mathrm{GeV}$, storage ring (Taiwan Light Source), operated at $300 \mathrm{~mA}$ electron current and with top-up injection. The photon energy of the unmonochromatized $X$-rays generated by this insertion device is $\approx 6.5-30 \mathrm{keV}$ and peaks at $\approx 12 \mathrm{keV}$. The $\mathrm{X}$-ray beams are trimmed by a pair of slits to obtain a transversal beam section $\approx 13 \mathrm{~mm} \times 9 \mathrm{~mm}$, to cover the complete precursor container. The dose rate was $4.7 \times 10^{5}$ Gy s$^{-1}$ (Wang et al., 2007; Liu et al., 2009). The relation between dose and flow rate is shown in Table 1. The temperature of the reaction chamber was controlled by cooling flows of compressed air aimed at the front and back windows. Fig. 1 outlines the continuous-flow Xray irradiation system.

\section{Table 1}

Relationship between flow rate, irradiation time and dose.

\begin{tabular}{llllllll}
\hline Flow rate $\left(\mathrm{ml} \mathrm{min} \mathrm{m}^{-1}\right)$ & 40 & 20 & 10 & 5 & 2 & 1 & 0.5 \\
Irradiation time (s) & 0.06 & 0.12 & 0.24 & 0.48 & 1.2 & 2.4 & 4.8 \\
Estimated dose (Gy) & $2.5 \times 10^{4}$ & $5.1 \times 10^{4}$ & $1.0 \times 10^{4}$ & $2.0 \times 10^{5}$ & $5.1 \times 10^{5}$ & $1.0 \times 10^{6}$ & $2.0 \times 10^{6}$
\end{tabular}

The fabricated nano-meshes were analyzed by transmission electron microscopy (TEM). The specimens for TEM were prepared by placing a drop of the solution on a carbon-coated copper grid and drying it at $40^{\circ} \mathrm{C}$. TEM measurements were performed in a JEM-2100F system operating at $200 \mathrm{kV}$.

Ultraviolet-visible (UV-Vis) spectra of the irradiated solution were acquired with an Ocean Optics USB4000 spectrophotometer with a $1 \mathrm{~cm}$ length cuvette. In addition to TEM 
characterization, scanning electron microscopy (SEM) images were recorded using a FEI Inspect F SEM.

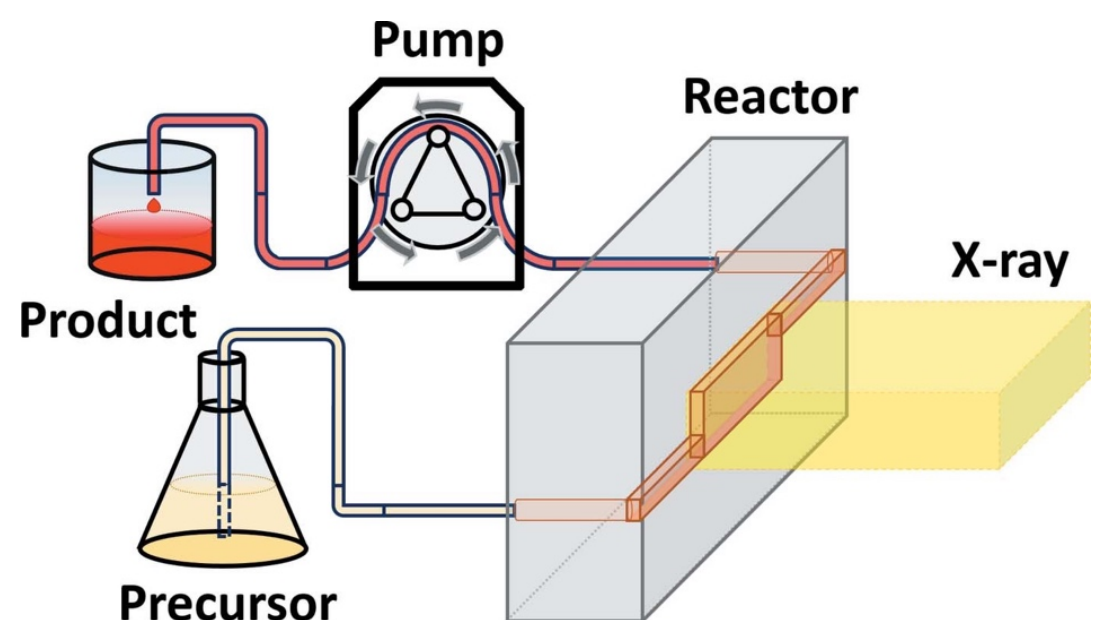

Figure 1

Schematic diagram of our continuous X-ray irradiation system.

The sheet resistance of the dried film was measured with a four-point probe (Quatek, QTI5601TSR) for nine different specimen areas. UV-Vis spectra were also taken for the dried nano-mesh films, using the glass substrate as a reference.

To prepare the nano-meshes, $0.1 \%$ PEG (polyethylene glycol) 20k was added to the irradiated precursor solution and then centrifuged at 3000 r.p.m. for $10 \mathrm{~min}$. The upper portion of the solution was decanted, obtaining a precipitated black solution. The product was resupplied with d.d. water reaching a $0.2 \mathrm{ml}$ volume and then re-dispersed into water by pipette. Then, $0.05 \mathrm{ml}$ of $2 \mathrm{wt} \%$ HPMC were added and the resulting 'ink' was slowly stirred to mix it well.

This ink was used to fabricate a transparent conductive film. A $100 \mathrm{~mm} \times 100 \mathrm{~mm}, 1 \mathrm{~mm}-$ thick glass slide was the substrate. Before coating, the glass was cleaned with acetone, methanol and d.d. water, and treated with a glow discharge (EMS100X, USA) to increase its hydrophilic properties. The glass was then coated with the 'ink' using a Mayer rod (model RDS-11) to hold the substrate; the relative speed of the rod with respect to the substrate was $5 \mathrm{~mm} \mathrm{~s}^{-1}$. The ink-coated glass was dried in a $50^{\circ} \mathrm{C}$ oven.

\section{Results and discussion}

Fig. 2 shows the UV-Vis absorption spectra of the Au precursor after irradiation for different flow rates. The inset shows the corresponding solutions. The smallest rates, $0.5,1$ and $2 \mathrm{ml}$ $\mathrm{min}^{-1}$, produced similar surface plasmon resonance (SPR) peaks. When the rate increased to $5 \mathrm{ml} \mathrm{min}-1$, the SPR started to shift and to become wider, revealing changes in the nanoparticle size and size distribution (Foss et al., 1994). For $10 \mathrm{ml} \mathrm{min}^{-1}$, the SPR disappeared and the solution color became dark gray. For additional increases of the rate to 20 and $40 \mathrm{ml} \mathrm{min}^{-1}$, the absorbance value decreased.

Fig. 3(a) shows representative TEM micrographs of Au nanoparticles in the irradiated solution. The size for a $1 \mathrm{ml} \mathrm{min}^{-1}$ flow rate was $7.5 \pm 1.9 \mathrm{~nm}$. When the flow rate increased to $5 \mathrm{ml} \mathrm{min}^{-1}$, the solution contained spherical Au nanoclusters with $13.4 \pm 3 \mathrm{~nm}$ diameter and some irregular chain-like segments, Fig. 3(b). At $10 \mathrm{ml} \mathrm{min}^{-1}$, the solution color became 
darker and the chain segments became longer, forming networks of Au nanowires with 6.6 $\pm 1.2 \mathrm{~nm}$ diameter, Fig. 3(c). The size of the networks, i.e. of the mesh structures, ranged from hundreds of nanometers to a few micrometers.

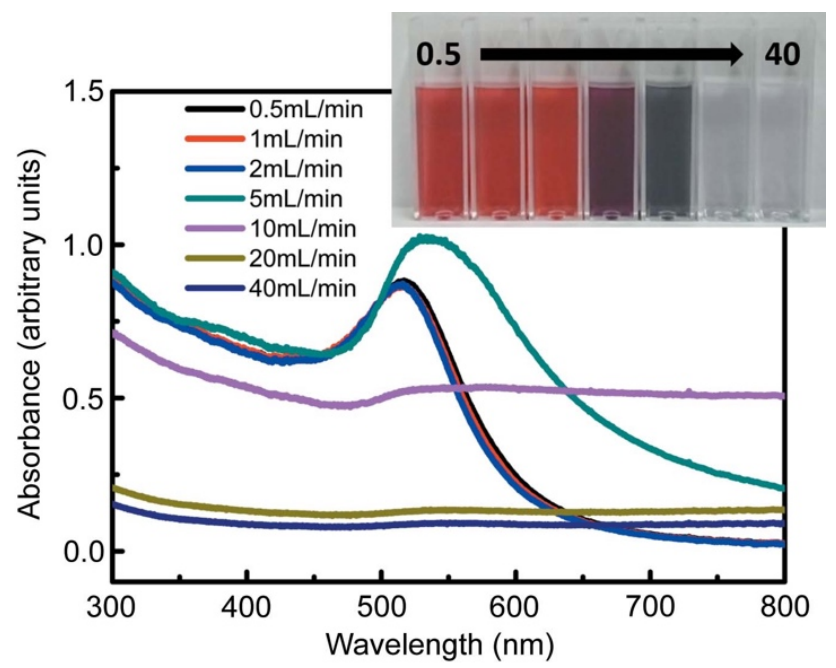

Figure 2

UV-Vis optical absorption spectra of our products for different flow rates. The visual appearance of the products is shown in the upper-right pictures.
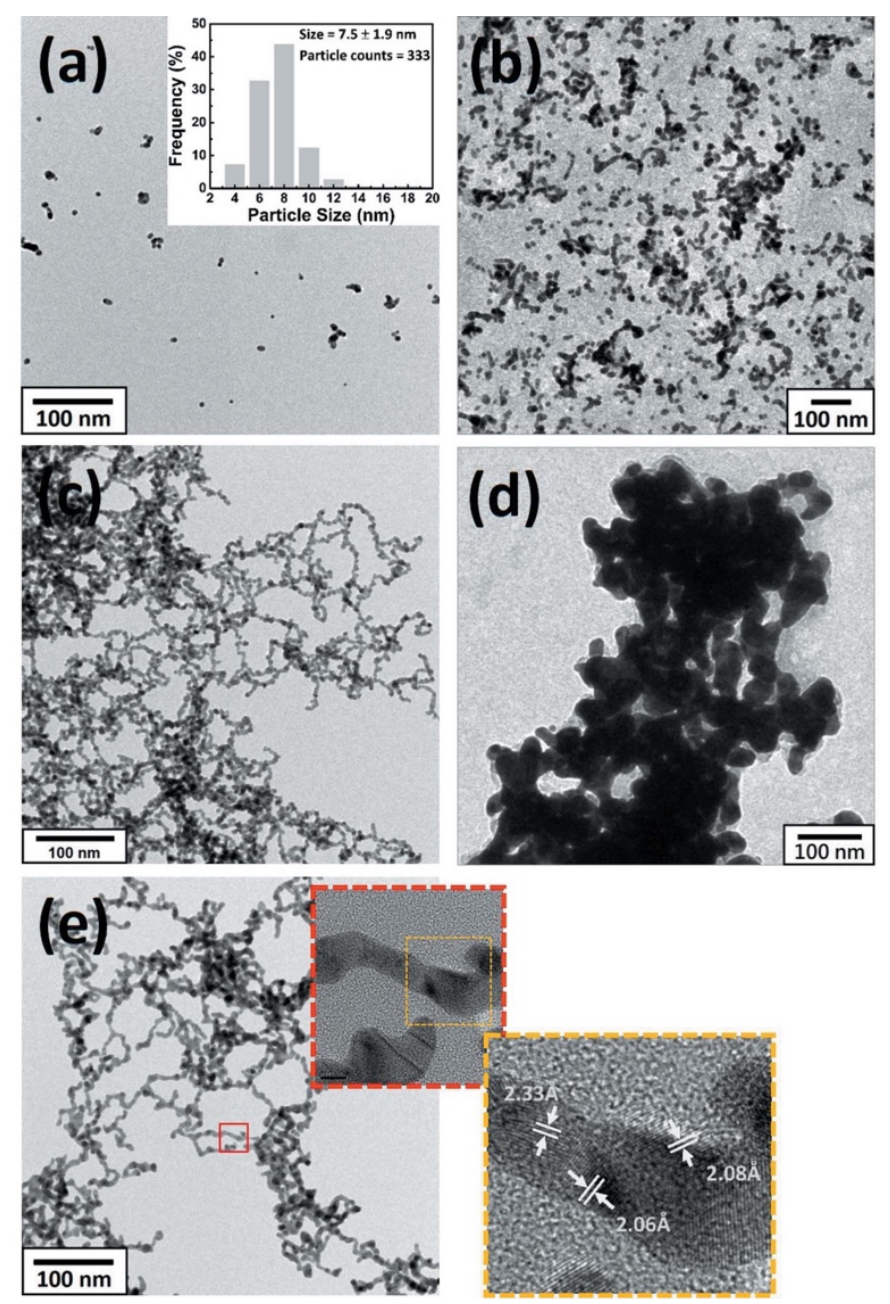

Figure 3

Typical TEM images of our dried products for different flow rates: 1, 5, 10, 20 and $40 \mathrm{ml} \mathrm{min}^{-1}$ for (a), (b), (c), (d) and (e). 
The high-resolution TEM images of Fig. 3(e) exhibit fringes attributed to diffraction from [111] and [200] planes, revealing a polycrystalline structure of the meshes (Pei et al., 2004). As the flow rate raised to $20 \mathrm{ml} \mathrm{min}^{-1}$, the nanoparticles fused into a big amorphous cluster, Fig. 3(d). In addition to gold nano-meshes obtained from the $1 \mathrm{mM}$ precursor, we also could obtain similar mesh structures from a $2 \mathrm{mM}$ Au precursor, but denser in mesh unit center and thicker in diameter (data not shown).

As mentioned, each flow rate gives a specific irradiation time: the above rates correspond to $0.06,0.12,0.24,0.48,1.2,2.4$ and $4.8 \mathrm{~s}$. The shortest time cannot produce a stable network nor well dispersed nanoparticles, hence the resulting big nanoparticle cluster. As the irradiation time increases, small nanoparticle clusters can assemble together along lines and produce large networks. For the longest irradiations the net structure breaks down, finally yielding stable isolated nano-spheres.

This trend is similar to that proposed by Pong et al. (2007) for Au nanoparticles produced by citrate reduction. Note, however, that our irradiation method does not require capping, which can prevent the production of conducting networks.

The effects of the solution $\mathrm{pH}$ are shown in Figs. 4 and 5, reporting UV-Vis and TEM results for mesh solutions obtained with a $10 \mathrm{ml} \mathrm{min}^{-1}$ flow rate. The $\mathrm{pH}$ was controlled by adding different amounts of $\mathrm{NaOH}$ to the precursor solution, obtaining $\mathrm{pH}$ values of 11.22, 10.35, 8.73, 6.21 and 4.3. We see in Fig. 4 that for a pH above 10 the Au SPR peak is weak, indicating the formation of nano-meshes. This is corroborated by the TEM images of Figs. $5(\mathrm{a})$ and $5(\mathrm{~b})$. As the $\mathrm{pH}$ value decreases, the SPR peak becomes more prominent; as confirmed by the TEM image of Fig. 5(c), this means that mesh structures are still formed, but with smaller sizes. Also, the majority of nanostructures are short segments of nanowires. This trend continues as shown by Fig. 5(d), with more short segments and quite a lot of spherical nanoparticles of size $19 \pm 3.4 \mathrm{~nm}$. For the lowest $\mathrm{pH}$ value, the nano-mesh structure completely disappears and is replaced by spherical nanoparticles of size $16.2 \pm$ $4.5 \mathrm{~nm}$.

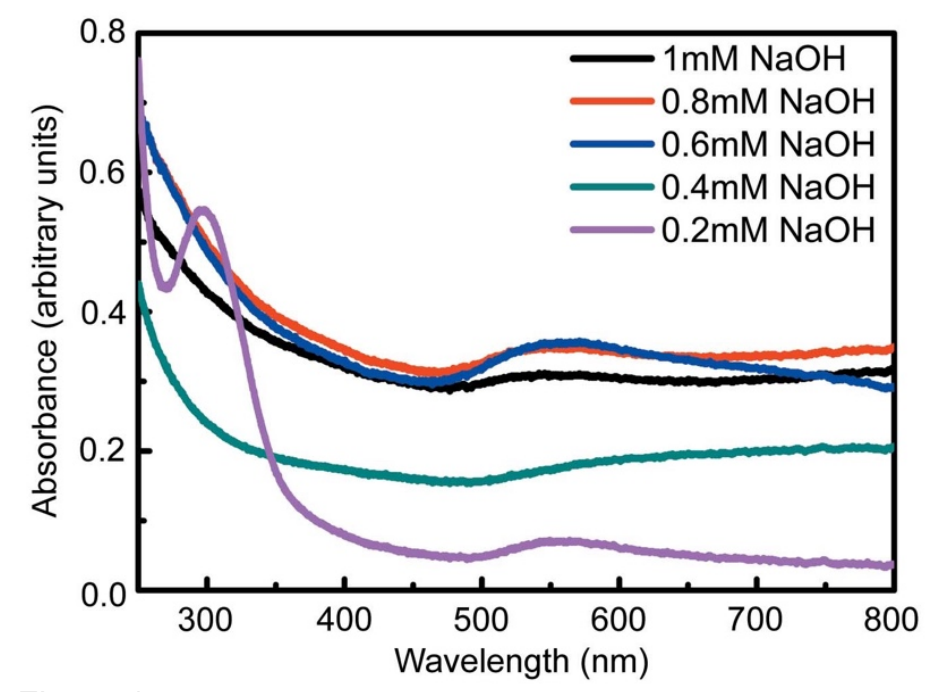

Figure 4

UV-Vis optical absorption spectra for a flow rate of $10 \mathrm{ml} \mathrm{min}^{-1}$ and for different $\mathrm{pH}$ values.

We discuss now the issue of capping. Capping agents are often added to the precursor solution to control the nanoparticles size and surface properties. For example, in the popular citrate reduction method of preparing gold nanoparticles the citrate ions act both as reducing 
agent and as stabilizing agent. In our previous X-ray synthesis methods, reduction was guaranteed by the solvated electron $\mathrm{e}^{-}$aq and by $\mathrm{H}^{\cdot}$ induced by X-rays (Wang et al., 2007), and stabilization of the Au nanoparticles by $\mathrm{pH}$ control. This means that our products were free of capping agents.

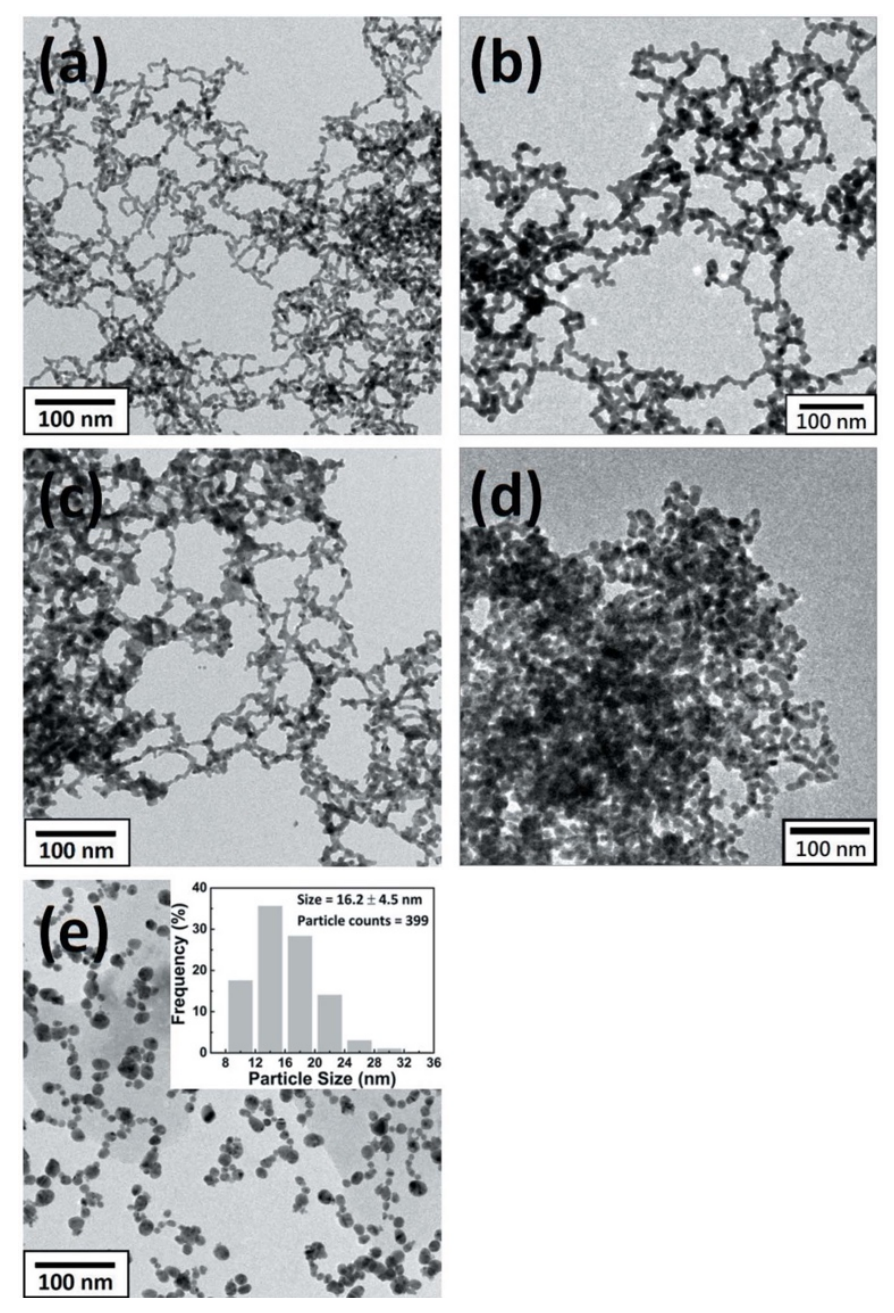

Figure 5

Typical TEM micrographs with $10 \mathrm{ml} \mathrm{min}^{-1}$ flow rate of flow system synthesis for different $\mathrm{NaOH}$ concentrations. (a) $1 \mathrm{mM}$, (b) $0.8 \mathrm{mM}$, (c) $0.6 \mathrm{mM}$, (d) $0.4 \mathrm{mM}$, (e) $0.2 \mathrm{mM}$.

Can the same approach be used for the fabrication of nano-meshes discussed here? We analyzed this question by adding different concentrations of the capping agent PVP (polyvinyl pyrrolidone) to the precursor solution while using a flow rate of $10 \mathrm{ml} \mathrm{min} \mathrm{m}^{-1}$. The results are shown in Fig. 6: higher capping agent concentrations lead to the formation of spherical particles.

We also tested low concentrations of different capping agents: PEG 6K, PVP 40K, PVA [poly(vinyl alcohol)] 130K, PEl (polyethylenimine) 2K, PAA [poly(acrylic acid)] 15K and citrate acid. The results are shown in Fig. 7: PEG, PVP and PVA give similar results, i.e. naked Au nano-meshes (data not shown). The addition of PEI, PAA or citrate acid breaks down the nano-mesh structure, and particles and nanowire fragments are detected. In summary, capping agents appear unnecessary or even detrimental for our present fabrication technique for TCEs. 
Finally, we tested the use of our meshes to realize trans- parent conductive thin films on 10 $\mathrm{cm} \times 10 \mathrm{~cm}$ glass substrates. Specific volumes of Au nano-meshes were collected by centrifugation. Then, HPMC was added as adhesive agent and DMSO was used to decrease de-wetting.

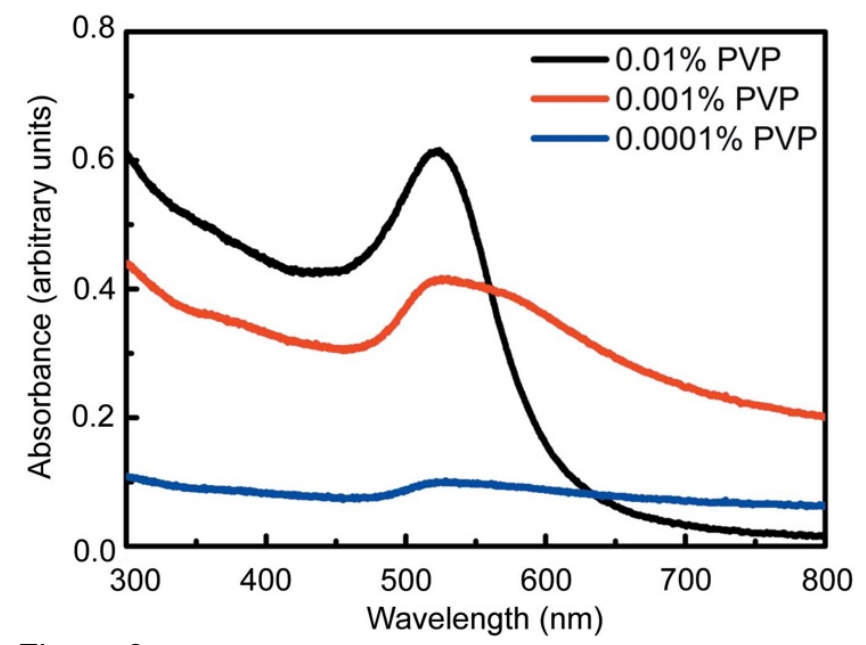

Figure 6

UV-Vis optical absorption spectra of products obtained with a $10 \mathrm{ml} \mathrm{min}^{-1}$ flow rate in the presence of different concentrations of the capping agent PVP.
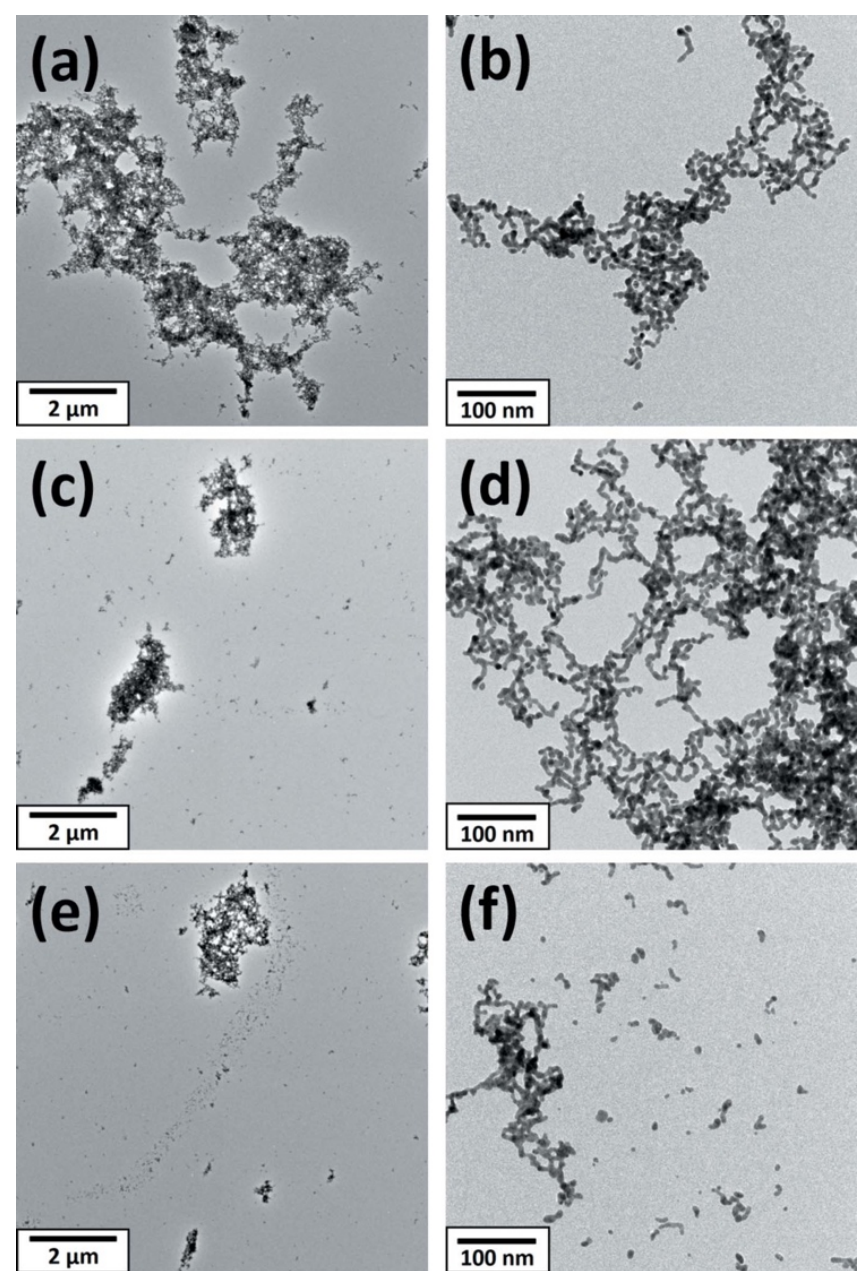

\section{Figure 7}

Typical TEM micrographs with two different magnifications of products obtained with a $10 \mathrm{ml} \mathrm{min}^{-1}$ flow rate in the presence of different capping agents: (a), (b) for PEI; (c), (d) for PAA; (e), (f) for citric acid. 
The results of sheet resistance and transmittance measurements on the resulting thin films are shown in Fig. 8. The lowest sheet resistance, $22.5 \Omega \mathrm{sq}^{-1}$ (over the $10 \mathrm{~mm} \times 10 \mathrm{~mm}$ coated area on glass substrate), was obtained with a $63.03 \mathrm{mg} \mathrm{ml}^{-1}(320 \mathrm{mM})$ Au nanomesh concentration, which had $34 \%$ transmittance. The highest transmittance, $58 \%$, was obtained with $31.51 \mathrm{mg} \mathrm{ml}^{-1}(160 \mathrm{mM})$, corresponding to a sheet resistance of $280 \Omega \mathrm{sq}^{-1}$. The UV-Vis transmission spectra of the films is featureless in the visible range. The SEM image of Fig. 9 shows the morphology of the transparent conductive film. The atomic force microscopy (AFM) image of Fig. 10 shows more details about the surface roughness.

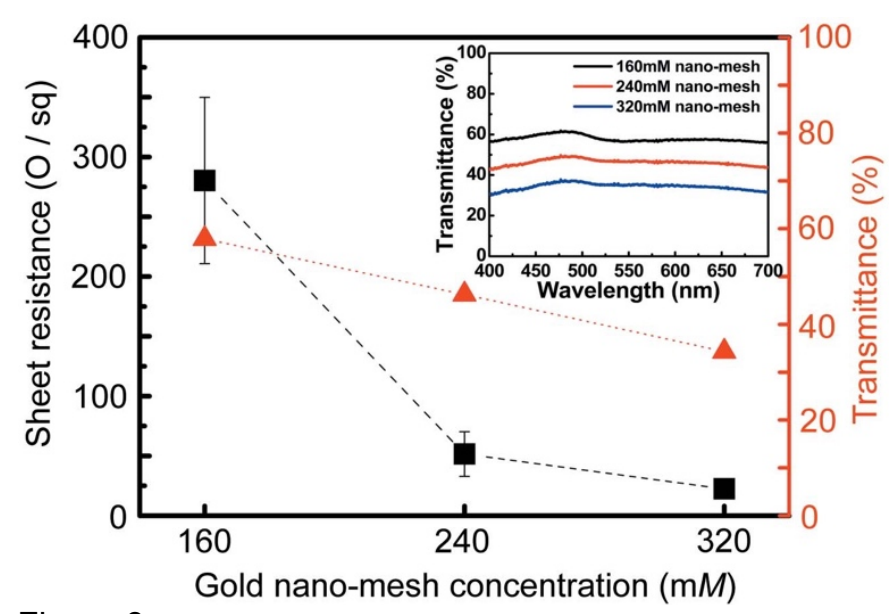

Figure 8

Sheet resistance and transmittance of conductive film for different Au nano-mesh concentrations. Inset: transmittance spectra in the visible for three concentrations.

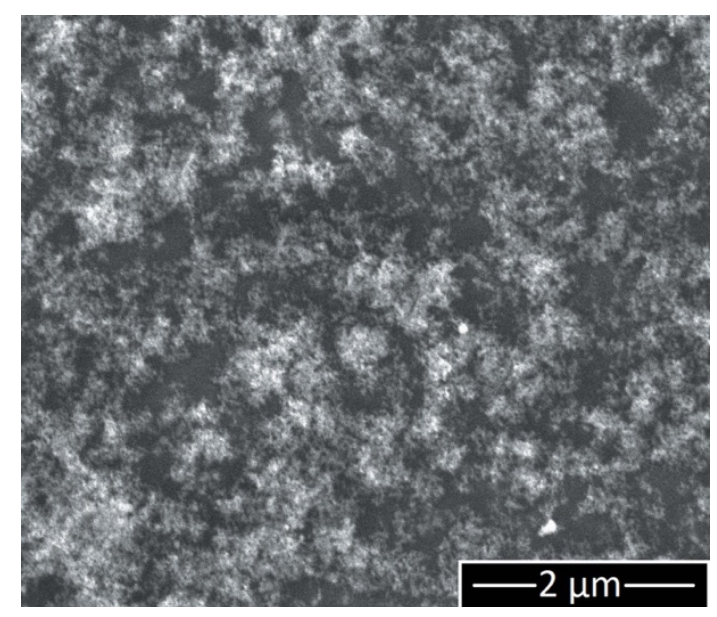

Figure 9

SEM image of one of our conductive films.

We can see some aggregated meshes that could explain the relatively high sheet resistance and low transmittance compared with the commercial ITO glass with transmittance $>80 \%$ and sheet resistance $<15 \Omega \mathrm{sq}^{-1}$ (Lee et al., 2008). Better synthesis control with the flow irradiation system is expected to sharply reduce or eliminate the aggregation. On the other hand, our irradiation method can use a wide range of synthesis parameters to control the Au wires thickness and length. A similar morphology tuning of silver nanowires, graphene and carbon nanotubes was very effective in increasing the transmittance and sheet conductance (Lee et al., 2013; Han et al., 2018; Farbod et al., 2017). 

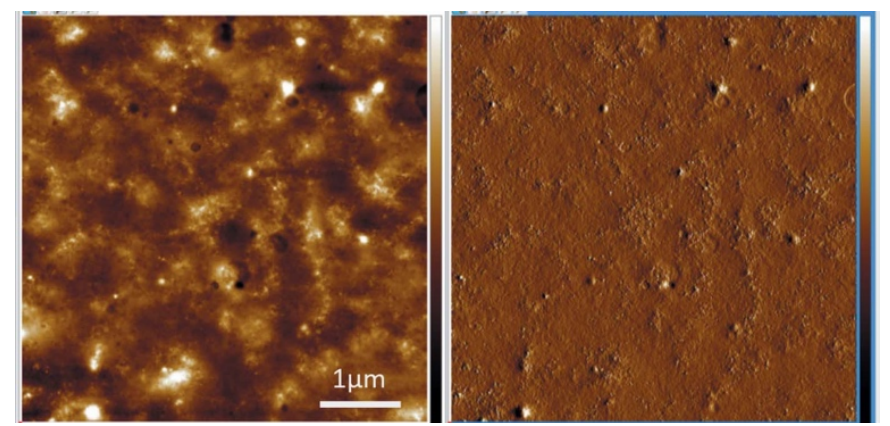

Figure 10

AFM image of the same specimen as Fig. 9.

Also note that, compared with other TCE films in their early development phase, e.g. $10^{2}$ to

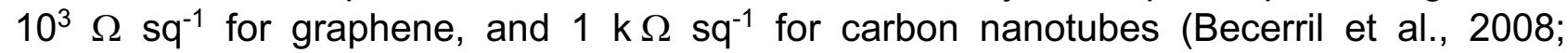
Kaempgen et al., 2005), the measured values of our nano-mesh Au TCE films are quite encouraging. The procedure is far from being optimized but even the preliminary tests give promising values. Therefore, the final objective of surpassing the above performances of commercial devices appears feasible.

For Ag nanowires prepared by polyol method, PVP usually acts as a structure-directing agent and capping agent. Together with PVP, other organic compounds, like isopropanol, also become trapped between the nanowires. This creates an insulating interface between the nanowires. These thin organic layers are difficult to remove unless they are heated up to their decomposition temperature (Langley et al., 2014). Also, high- temperature processes inhibit the application of temperature- sensitive substrate. Our gold nano-mesh is a capping-agent- free material, and will benefit from its natural formation.

\section{Conclusions}

We have developed a continuous-flow X-ray irradiation technique that offers precise control of the irradiation dose, elimination of convection effects in the precursor solution, and suitability for large-scale production. The Au nano-meshes of interest as transparent and flexible electrodes for optoelectronics were fabricated by this method; the synthesis parameters and the product properties were extensively characterized, with rather encouraging results.

\section{Acknowledgements}

We thank Stephanie Hwu for the graphic works.

\section{References}

Becerril, H. A., Mao, J., Liu, Z., Stoltenberg, R. M., Bao, Z. \& Chen, Y. (2008). ACS Nano, 2, 463-470.

Catrysse, P. B. \& Fan, S. (2010). Nano Lett. 10, 2944-2949.

Chen, Z., Cotterell, B. \& Wang, W. (2002). Eng. Fract. Mech. 69, 597-603.

Cheon, Y., Nam, J., Kim, C. S. \& Jo, S. (2017). AIP Adv. 7, 025215.

De, S., Higgins, T. M., Lyons, P. E., Doherty, E. M., Nirmalraj, P. N., Blau, W. J., Boland, J. J. \& Coleman, J. N. (2009). ACS Nano, 3, 1767-1774. 
Doherty, E. M., De, S., Lyons, P. E., Shmeliov, A., Nirmalraj, P. N., Scardaci, V., Joimel, J., Blau, W. J., Boland, J. J. \& Coleman, J. N. (2009). Carbon, 47, 2466-2473.

Eda, G., Fanchini, G. \& Chhowalla, M. (2008). Nat. Nanotechnol. 3, 270-274.

Edwards, P. P., Porch, A., Jones, M. O., Morgan, D. V. \& Perks, R. M. (2004). Dalton Trans. pp. 2995-3002.

Farbod, M., Zilaie, A. \& Kazeminezhad, I. (2017). J. Sci. Adv. Mater. Dev. 2, 99-104.

Foss, C. A., Hornyak, G. L., Stockert, J. A. \& Martin, C. R. (1994). J. Phys. Chem. 98, 2963-2971. Gou, L., Chipara, M. \& Zaleski, J. M. (2007). Chem. Mater. 19, 1755-1760.

Han, N. M., Wang, Z., Shen, X., Wu, Y., Liu, X., Zheng, Q., Kim, T.-H., Yang, J. \& Kim, J.-K. (2018). ACS Appl. Mater. Interfaces, 10, 6580-6592.

Hecht, D. S., Hu, L. \& Irvin, G. (2011). Adv. Mater. 23, 1482-1513.

Kaempgen, M., Duesberg, G. S. \& Roth, S. (2005). Appl. Surf. Sci. 252, 425-429.

Kang, M. G. \& Guo, L. J. (2007). Adv. Mater. 19, 1391-1396. Khaligh, H. H. \& Goldthorpe, I. A. (2013). Nanoscale Res. Lett. 8, 235.

Lai, S. F., Chien, C. C., Chen, W. C., Chen, H. H., Chen, Y. Y., Wang, C. L., Hwu, Y., Yang, C. S., Chen, C. Y., Liang, K. S., Petibois, C., Tan, H. R., Tok, E. S. \& Margaritondo, G. (2013). Biotechnol. Adv. 31, 362-368. Lai, S.-F., Chien, C.-C., Chen, W.-C., Chen, Y.-Y., Wang, C.-H., Hwu, Y., Yang, C. S. \& Margaritondo, G. (2012). RSC Adv. 2, 6185-6191.

Lai, S. F., Tan, H. R., Tok, E. S., Chen, Y. H., Ong, E. B. L., Li, M. T., Chen, Y. Y., Chien, F. C., Chen, P., Margaritondo, G. \& Hwu, Y. (2015). Chem. Commun. 51, 7954-7957.

Langley, D. P., Lagrange, M., Giusti, G., Jime' nez, C., Bréchet, Y., Nguyen, N. D. \& Bellet, D. (2014). Nanoscale, 6, 13535-13543. Lee, J., Lee, P., Lee, H. B., Hong, S., Lee, I., Yeo, J., Lee, S. S., Kim, T.-S., Lee, D. \& Ko, S. H. (2013). Adv. Funct. Mater. 23, 4171-4176.

Lee, J. Y., Connor, S. T., Cui, Y. \& Peumans, P. (2008). Nano Lett. 8, 689-692.

Li, M. T., Wang, C. H., Lai, S. F., Ong, E. B. L., Chen, Y. H., Lin, C. K., Margaritondo, G. \& Hwu, Y. (2015). Nanotechnology, 26, 355601.

Liu, C.-J., Wang, C.-H., Wang, C.-L., Hwu, Y., Lin, C.-Y. \& Margaritondo, G. (2009). J. Synchrotron Rad. 16, 395-397.

Pei, L., Mori, K. \& Adachi, M. (2004). Langmuir, 20, 7837-7843. Pong, B. K., Elim, H. I., Chong, J. X., Ji, W., Trout, B. L. \& Lee, J. Y. (2007). J. Phys. Chem. C, 111, 6281-6287.

Reina, A., Jia, X., Ho, J., Nezich, D., Son, H., Bulovic, V., Dresselhaus, M. S. \& Kong, J. (2009). Nano Lett. 9 , 30-35.

Song, Y.-F., Chang, C.-H., Liu, C.-Y., Chang, S.-H., Jeng, U.-S., Lai, Y.-H., Liu, D.-G., Chung, S.-C., Tsang, K.-L., Yin, G.-C., Lee, J.-F., Sheu, H.-S., Tang, M.-T., Hwang, C.-S., Hwu, Y.-K. \& Liang, K. S. (2007). J. Synchrotron Rad. 14, 320-325.

Tung, V. C., Chen, L. M., Allen, M. J., Wassei, J. K., Nelson, K., Kaner, R. B. \& Yang, Y. (2009). Nano Lett. 9, 1949-1955.

Wang, C.-H., Chien, C.-C., Yu, Y.-L., Liu, C.-J., Lee, C.-F., Chen, C.-H., Hwu, Y., Yang, C.-S., Je, J.-H. \& Margaritondo, G. (2007). J. Synchrotron Rad. 14, 477-482.

Wang, C. H., Liu, C. J., Wang, C. L., Chien, C. C., Hwu, Y., Liu, R. S., Yang, C. S., Je, J. H., Lin, H. M. \& Margaritondo, G. (2009). Appl. Phys. A, 97, 295-300.

Wang, C. H., Liu, C. J., Wang, C. L., Hua, T. E., Obliosca, J. M., Lee, K. H., Hwu, Y., Yang, C., Liu, R. S., Lin, H. M., Je, J. H. \& Margaritondo, G. (2008). J. Phys. D Appl. Phys. 41, 195301.

Wang, C. L., Hsao, B. J., Lai, S. F., Chen, W. C., Chen, H. H., Chen, Y. Y. C., Chien, C. C., Cai, X., Kempson, I. M., Hwu, Y. \& Margaritondo, G. (2011). Nanotechnology, 22, 065605.

Wu, Z., Chen, Z., Du, X., Logan, J. M., Sippel, J., Nikolou, M., Kamaras, K., Reynolds, J. R., Tanner, D. B., Hebard, A. F. \& Rinzler, A. G. (2004). Science, 305, 1273-1276.

Yang, Y.-C., Wang, C.-H., Hwu, Y.-K. \& Je, J.-H. (2006). Mater. Chem. Phys. 100, 72-76.

Zhang, P., Wyman, I., Hu, J., Lin, S., Zhong, Z., Tu, Y., Huang, Z. \& Wei, Y. (2017). Mater. Sci. Eng. B, 223, $1-23$. 